\title{
Interstitial Collagenase Is a Brownian Ratchet Driven by Proteolysis of Collagen
}

\author{
Saveez Saffarian, ${ }^{1}$ Ivan E. Collier, ${ }^{2}$ Barry L. Marmer, ${ }^{2}$ \\ Elliot L. Elson, ${ }^{1}$ Gregory Goldberg ${ }^{1,2 *}$
}

\begin{abstract}
We show that activated collagenase (MMP-1) moves processively on the collagen fibril. The mechanism of movement is a biased diffusion with the bias component dependent on the proteolysis of its substrate, not adenosine triphosphate (ATP) hydrolysis. Inactivation of the enzyme by a single amino acid residue substitution in the active center eliminates the bias without noticeable effect on rate of diffusion. Monte Carlo simulations using a model similar to a "burnt bridge" Brownian ratchet accurately describe our experimental results and previous observations on kinetics of collagen digestion. The biological implications of MMP-1 acting as a molecular ratchet tethered to the cell surface suggest new mechanisms for its role in tissue remodeling and cell-matrix interaction.
\end{abstract}

The extracellular matrix (ECM) of vertebrates is a three-dimensional scaffold consisting of highly organized macromolecular assemblies that vary in structure and composition to define and maintain the shapes and mechanical properties of tissues. Many physiological and pathophysiological processes from morphogenesis to wound healing, tumor progression, and metastatic invasion are characterized by intensified tissue remodeling that begins with degradation of the existing $\operatorname{ECM}(1,2)$.

Collagen is the most abundant component of the ECM. Monomers of fibrillar collagens have a unique triple-helical structure that self-assembles to produce tightly packed periodic fibrils (3). Fibrils can be up to $500 \mathrm{~nm}$ in diameter and are highly resistant to proteolytic degradation. Resident cells of tissues can secrete a specialized group of enzymes, matrix metalloproteases (MMPs), that degrade ECM macromolecules including collagens (4). Interstitial collagenase, MMP-1 $(5,6)$, cleaves all three $\alpha$ chains of the collagen monomer at a single site. The assembled collagen fibril contains multiple cleavage sites spaced $300 \mathrm{~nm}$ apart. Here, we demonstrate that the digestion of a collagen fibril occurs when the bound MMP-1 undergoes biased diffusion along the fibril and encounters cleavage sites without noticeable dissociation. The MMP-1 transport mechanism is akin to a Brownian ratchet with biased diffusion independent of ATP hydrolysis but coupled to collagen proteolysis instead.

${ }^{1}$ Department of Biochemistry and Molecular Biophysics and ${ }^{2}$ Division of Dermatology, Washington University School of Medicine, St. Louis, MO 63110, USA.

${ }^{*}$ To whom correspondence should be addressed. E-mail: goldberg@medicine.wustl.edu
Feynman showed (7) that particles diffusing in an anisotropic environment cannot produce work in an isothermal system, but a thermal gradient applied to the same system can bias the diffusion. This makes microscopic machines plausible. There are now several models of biased diffusion that do not require a system-wide gradient or field (8), among them Brownian ratchets (9-12). In a "burnt bridge" model of a Brownian ratchet (13), the diffusion bias is created because the moving particle destroys weak places on its track, so its ability to diffuse back is inhibited. A slight modification of the burnt bridge model explains our observation of MMP-1 activity.

We used two-photon excitation fluorescence correlation spectroscopy (FCS) to observe the interaction of MMP-1 molecules with collagen fibrils in their most native state in a hydrated collagen gel. Under these conditions, the substrate is resistant to other nonspecific proteases. Collagen gels with oriented fibrils were produced by polymerizing acid-soluble rat-tail collagen in a magnetic field of $8 \mathrm{~T}$ (14) and were decorated with fluorescent dye (Alexa 488)-labeled enzyme (15). A selected individual fibril was centered under the 5-mW laser beam and illuminated for $120 \mathrm{~s}$ to achieve a constant average fluorescence, indicating a steady state. The initial exposure was immediately followed by collection of primary fluorescence data for $300 \mathrm{~s}$ with a bin time of $400 \mu$ s (Fig. 1, A.1). We obtained the control record from undecorated fibrils by using collagen luminescence for imaging. The primary fluorescence data from both the experiment and the control were dominated by shot noise. Increasing the bin time of the primary $400-\mu \mathrm{s}$ record to $80 \mathrm{~ms}$ (Fig. 1, A.2) revealed large spikes of fluorescence present in the record obtained from MMP-1decorated fibrils but not in the control record. The appearance of these spikes was completely suppressed when the bound Alexa 488-labeled enzyme was immobilized by cross-linking to the fibrils before observation. The intensity of the spikes was well within the limits expected for a single molecule of labeled enzyme [Fig. 1, A.3, and (15)]. Because contribution of enzyme readsorption to the appearance of the spikes is insignificant (15), we conclude that the spikes of fluorescence intensity observed in FCS experiments on an individual MMP-1-decorated fibril represent single molecules of the collagen-bound enzyme passing through the laser beam (15).

To isolate the spikes from the background [see Spatial Background Filter (15)], we scanned the $80-\mathrm{ms}$ bin time record (Fig. 1, A.2) with a 2-s window. The fluorescence above the threshold set to five times the standard deviation of the average signal in each window was counted as a spike, and its position was recorded. This information was then used to suppress the background contribution and to calculate a correlation function (Fig. 1B) using the 400- $\mu$ s binned fluorescence record $(15,16)$. A particle diffusing freely in one dimension has a finite probability of returning to a point that it visited previously. Therefore, the correlation function characteristic of one-dimensional (1D) diffusion has an elongated tail. The experimental correlation function for the active enzyme (Fig. 1B) deviates from the free diffusion model (15) but fits well to a $1 \mathrm{D}$ diffusion plus flow model (17) in which particles exhibit diffusive behavior at fast time scales but have zero probability of return at longer times, owing to the directional flow. The local diffusion coefficient $D=8 \pm$ $1.5 \times 10^{-9} \mathrm{~cm}^{2} \mathrm{~s}^{-1}$ and the transport velocity $V=4.5 \pm 0.36 \mu \mathrm{m} \mathrm{s}^{-1}$ were determined from the fit of the correlation function obtained from the wild-type activated MMP-1.

Biased diffusion is a characteristic of a molecular motor and requires energy dissipation. For classical molecular motors, energy comes from ATP hydrolysis (18-20). In the absence of ATP, we hypothesized that energy may come from coupling to proteolysis. To investigate this possibility, we inactivated the enzyme by a single point mutation, glutamic acid at position 219 to glutamine (E219Q), in its active center (15). The mutation specifically eliminates the catalytic activity of the enzyme with little or no effect on enzyme structure or binding to collagen $(21,22)$. FCS measurements on the inactive MMP-1 mutant gave experimental correlation functions with an elongated tail consistent with 1D diffusion with no directional bias (Fig. 1B). The diffusion coefficient $D=6.7 \pm 1.5 \times 10^{9} \mathrm{~cm}^{2} \mathrm{~s}^{-1}$ 
was similar to the diffusion coefficient of the active enzyme; this finding suggests that the inactivation of the enzyme has little effect on the diffusion properties of MMP-1 on the collagen fibrils and that the transport mechanism depends on the catalytic activity of the enzyme. The values of the diffusion coefficient obtained for wild-type and mutant MMP-1 on collagen fibril are in good agreement with those for MMP-2 proenzyme on a gelatin layer that we determined earlier (23).

To verify these results and to determine whether the biased component dominates the transport process on a macroscopic scale, we measured the flux of single MMP-1 molecules around a "no-transport" block created on a collagen fibril (Fig. 2). When MMP-1decorated collagen fibrils are photobleached

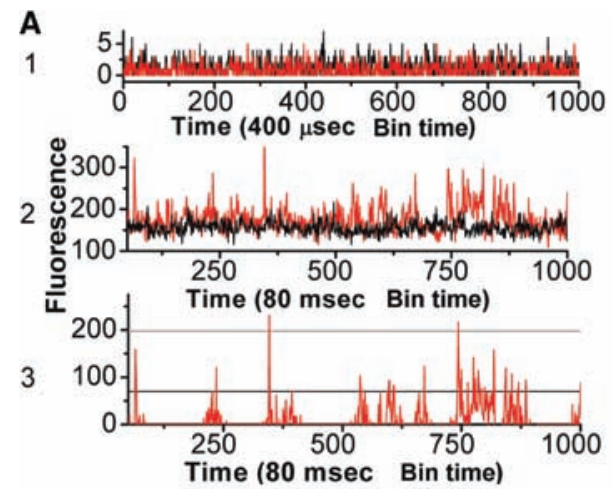

B

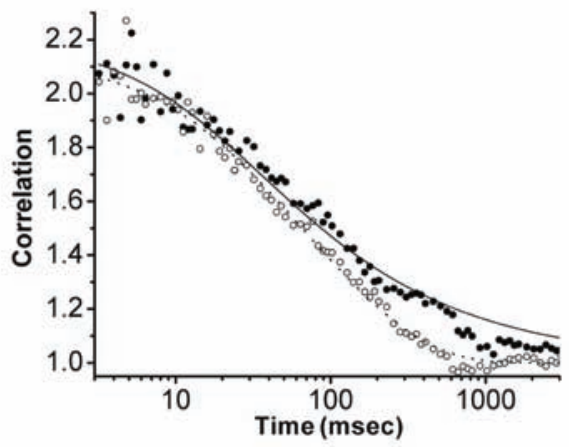

Fig. 1. Mobility of MMP-1 bound to a collagen fibril. (A) An individual collagen fibril was positioned under the laser beam by using a nano positioning stage. A constant average fluorescence indicating a steady state was achieved in the first $120 \mathrm{~s}$, after which fluorescent signal was collected for $300 \mathrm{~s}$ with a 400- $\mathrm{s}$ bin time. (A.1) Primary fluorescent record of an MMP-1decorated (red) and an untreated collagen fibril (black) recorded at a $400-\mu$ s bin time and plotted as the number of collected photons versus the bin number. One thousand bins out of the entire 300-s experiment are shown. Both records are dominated by shot noise. (A.2) Primary fluorescent count record as in A.1 displayed with the bin time increased to $80 \mathrm{~ms}$. Spikes of fluorescent counts are observed in the experiments performed on MMP-1-decorated fibrils. Background fluorescence from untreated collagen fibrils shows no spikes in the record. (A.3) The 80 -ms binned record from A.2 was used for finding the position of the spikes (15). Isolated from the background, the spikes of fluorescence intensity represent single molecules of MMP-1 passing through the laser beam. The single-molecule fluorescence was measured in FCS experiments with Alexa 488-labeled MMP-1 in solution. The average value of $870 \mathrm{~Hz}$ (black line) and a maximum of $2.4 \mathrm{kHz}$ (gray line) were calculated either by averaging the fluorescence over the entire observation volume or by assuming that the molecule traveled through the center of the 300-nm waist beam, respectively. The intensity of spikes is within the expected range for a single-molecule intensity. (B) The normalized experimental correlation functions were calculated by using the $400-\mu$ s binned records from six independent experiments. The background was suppressed using the spatial background filter (15). The correlation function obtained from collagen fibrils decorated with either wild-type activated MMP-1 (open circles) or its inactive mutant (filled circles). The experimental correlation function for wild-type activated enzyme was fitted (gray line) to a flow plus diffusion model (17)

$$
G(t)=1+\exp \left[-\left(t / \tau_{f}\right)^{2} \frac{1}{1+t / \tau_{D}}\right] \frac{1}{\left(1+t / \tau_{D}\right)^{0.5}}
$$

where $\tau_{D}=w^{2} / 4 D$ is the diffusion time, $\tau_{f}=w / V$ is the flow time, $w$ is the beam waist, $D$ is the diffusion coefficient and $V$ is the flow velocity. The shown fit of the wild-type MMP-1 data has a diffusion coefficient of $8 \pm 1.5 \times 10^{-9} \mathrm{~cm}^{2} \mathrm{~s}^{-1}$ and a transport velocity of $4.5 \pm 0.36 \mu \mathrm{m} \mathrm{s}^{-1}$. The correlation function of the inactive mutant exhibits a long tail characteristic of an unbiased 1-D diffusion $G(\tau)=1+\left[1 /\left(1+t / \tau_{D}\right)^{0.5}\right]$, with a local diffusion coefficient of $6.7 \pm 1.5 \times$ $10^{-9} \mathrm{~cm}^{2} \mathrm{~s}^{-1}$. zyme, whereas the inactive mutant shows complete symmetry (Fig. 2). Intermediate results were observed when proteolysis was inhibited in the presence of the metalloprotease inhibitor, Galardin, or the collagen fibrils were immersed in heavy water (25). Thus, the active MMP-1 bound to a collagen fibril undergoes proteolysis-dependent directional transport, with the degree of asymmetry in the flux depending on the efficiency of proteolysis.

What is the mechanism that rectifies symmetric diffusive motion to induce biased transport of the active enzyme along the fibril? We considered a theoretical model similar to a recently formalized burnt bridges Brownian ratchet (13), with two assumptions (Fig. 3): First, at recognition sites, a cleavage occurs with a probability $P_{\mathrm{C}}$ and the enzyme always ends up on a specific side of the cleaved peptide bond. Second, the molecules are not allowed to cross the cleaved collagen helix from either side but are allowed to

\section{MMP-1 Decorated Fibril with a Bleached "No Transport" Block}

\section{A. Nondirectional diffusion}

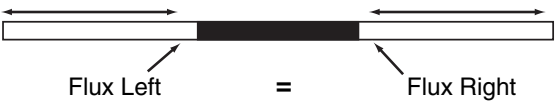

\section{B. Directional transport}

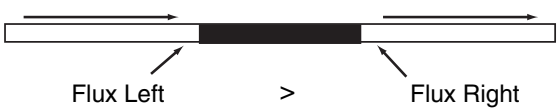

\section{Average Number of Molecules in:}

\begin{tabular}{lrrr}
\hline & Left & Right & Ratio \\
& Flux & Flux & \\
Activated WT & 44.5 & 5.5 & $0.81 \pm 0.1$ \\
Inactivated mutant & 50.6 & 42.3 & $0.1 \pm 0.06$ \\
Heavy water & 34.5 & 14.5 & $0.39 \pm 0.08$ \\
Galardin & 77.6 & 32 & $0.44 \pm 0.08$
\end{tabular}

Fig. 2. The unequal flux of MMP-1 molecules around a no-transport block on the collagen fibril. (A and B) Photobleaching of MMP-1decorated collagen fibrils (white bar) with a laser beam intensity below $50 \mathrm{~mW}$ results in the recovery of fluorescence after termination of beam exposure (24). Raising the laser intensity to 80 to $90 \mathrm{~mW}$ prevents recovery after photobleaching, which indicates a blockage of the enzyme transport across the bleached area (solid bar) due to a damage of the fibril. (C) The average number of single enzyme molecules passing through the laser beam in the left $\left\langle C_{L}\right\rangle$ and right $\left\langle C_{R}\right\rangle$ flanks of the no-transport block are calculated by counting the number of spikes in the fluorescence record and are averaged from three independent experiments for each form of the enzyme. The differences are expressed as the asymmetry ratio calculated as $\left\langle C_{L}-C_{R} / C_{L}+C_{R}\right\rangle$, which is then averaged over all three experiments so that 1 indicates a perfect asymmetry and 0 a completely symmetric experiment. The arrows indicate the direction of enzyme transport. 


\section{R E P O R T S}

jump to a neighboring triple-helical track with a small probability $P_{\mathrm{J}}$. This mechanism produces a net transport (13), with velocity $V$ that depends on the diffusion coefficient, the probability $P_{\mathrm{C}}$, and the spatial distribution of the cleavage sites along the tracks (300 $\mathrm{nm}$ apart for a collagen fibril).

The analytical solution of this model for an enzyme molecule moving on a single track with an equidistant distribution of sites and low $P_{\mathrm{C}}$ is given in (13). To model the behavior of an ensemble of the enzyme molecules diffusing on a fibril, we have constructed Monte Carlo simulations as described in [Fig. 3B and (15)]. The simulations
(Fig. 4) demonstrate that both correlation functions and asymmetry ratios are dependent on $P_{\mathrm{C}}$, whereas the rate of jumping to a neighboring triple-helical track $P_{\mathrm{J}}=1 / 200 \mathrm{~s}$ remains constant (fig. S2). With the experimentally determined diffusion coefficient $\left(D=8 \pm 1.5 \times 10^{-9} \mathrm{~cm}^{2} \mathrm{~s}^{-1}\right)$ and a Gaussian laser profile with a waist of $300 \mathrm{~nm}$, simulations of correlation functions and the asymmetry ratios (Fig. 4) reproduced those obtained experimentally for the active enzyme when $P_{\mathrm{C}}=10 \%$. Similarly, change of a value of a single parameter, $P_{\mathrm{C}}=2 \times 10^{-5}$, in simulations reproduced experimental data for the mutant enzyme (15). The asymmetry
Fig. 3. Burnt bridges mechanism of MMP-1 transport on a collagen fibril. (A) The rules of the road. The enzyme molecules perform a random walk (curved open arrows) in one-dimension along a collagen fibril. Once they reach a cleavage recognition site (circles), a successful cleavage (open circles) occurs with a set probability $P_{C}$, and the enzyme molecule responsible for the cleavage always ends up on one specific side of the cleaved peptide bond. The molecules are not allowed to cross the cleaved triple helix from either side
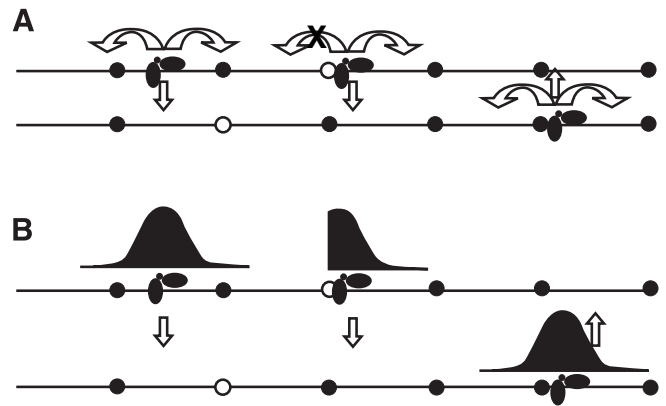

but are allowed to jump to a neighboring triple-helical track with a small probability $P_{\jmath}$ (vertical arrows). This mechanism, akin to a Brownian ratchet, produces a net transport with velocity $V$, which depends on a diffusion coefficient, the probabilities defined above and the spatial distribution of the cleavage sites. (B) Monte Carlo simulations of the interaction of MMP-1 with a collagen fibril. To construct a Monte Carlo simulation of MMP-1 motion on collagen, we monitored 10 enzyme molecules on a 30- $\mu$ m-long fibril with a cross section of 100 triple helixes of collagen and cleavage sites $0.3 \mu \mathrm{m}$ apart. In the beginning of an experiment, MMP- 1 molecules were positioned randomly on a fibril and were allowed to diffuse according to the rules described above. The value of $P$, was set at $1 / 200 \mathrm{~s}$, and the diffusion coefficient was as determined experimentally. Once molecules exited the monitored $30-\mu \mathrm{m}$ region they were put back on the opposite end of the fibril to approximate the conditions on a long fibril. The random walk steps have a Gaussian size distribution and walkers encounter a reflective boundary condition at each of the burnt bridges (open circles). In each case, the results of three Monte Carlo simulation runs with different seeds were averaged. ratio has an exponential dependence on the probability of cleavage $P_{\mathrm{C}}$ so that a $50 \%$ decline in the asymmetry ratio corresponds to a two orders of magnitude drop in the cleavage probability. Because correlation functions and the asymmetry ratios are experimentally independent, it is notable that Monte Carlo simulations accurately predict both for active and inhibited enzymes, depending on the value of a single parameter $P_{\mathrm{C}}$.

Because cleavage of a triple-helical track by a passing enzyme acts as a roadblock for molecules following on the same track, the model predicts the accumulation of enzyme molecules confined to a free diffusion between cleaved sites, which create a traffic jam. Relief of the traffic jam is achieved by the transfer of the trapped enzyme to new tracks with probability $P_{\mathrm{J}}$. In the computer simulations where $P_{\mathrm{J}}=1 / 200 \mathrm{~s}^{-1}$ and $P_{\mathrm{C}}=$ $10 \%$, the traffic jam is seen as peaks of the enzyme concentration along the fibril that disappear when $P_{\mathrm{C}}=0$ (Fig. $4 \mathrm{~A}$, inset). In our experiments, the trapped enzyme is bleached during the first $120 \mathrm{~s}$ of beam exposure. The traffic jam phenomenon also explains certain peculiarities of the collagen digestion kinetics observed earlier (26). In these experiments, the overall speed of collagen digestion increased linearly with the enzyme concentration. However, at a nonsaturating concentration of MMP-1, the enzyme activity rose substantially with an increase of collagen substrate concentration. Under conditions of the traffic jam, presenting more substrate reduces the average concentration of the trapped enzyme, which causes an increase in overall digestion rate.

Thermodynamic measurements support this interpretation. At a constant substrate concentration, the acquisition of the new tracks

Fig. 4. Monte Carlo simulations of the experimental correlation functions and the asymmetry ratio. (A) Monte Carlo simulations were performed as in Fig. 3B. Two simulated correlation functions for $P_{C}=10 \%$ (open circles) and $P_{c}=0$ (filled circles), each an average of three Monte Carlo simulation runs with different seeds, were fitted as in Fig. 1 with either 1D dif-

A

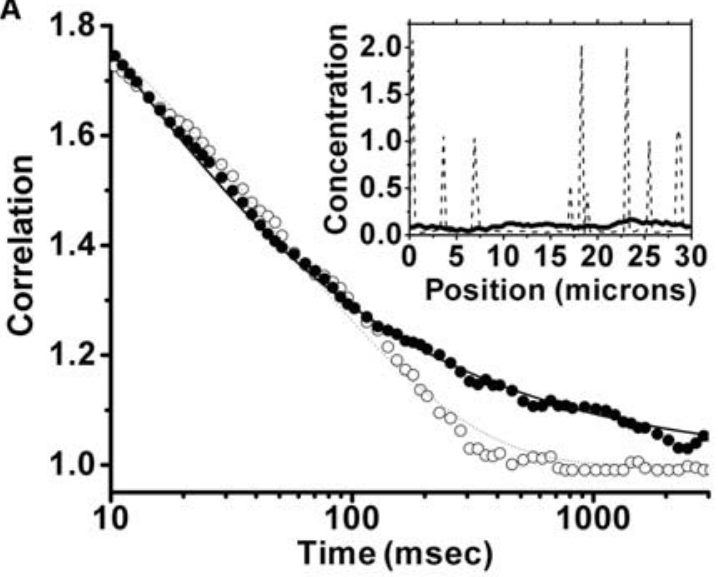

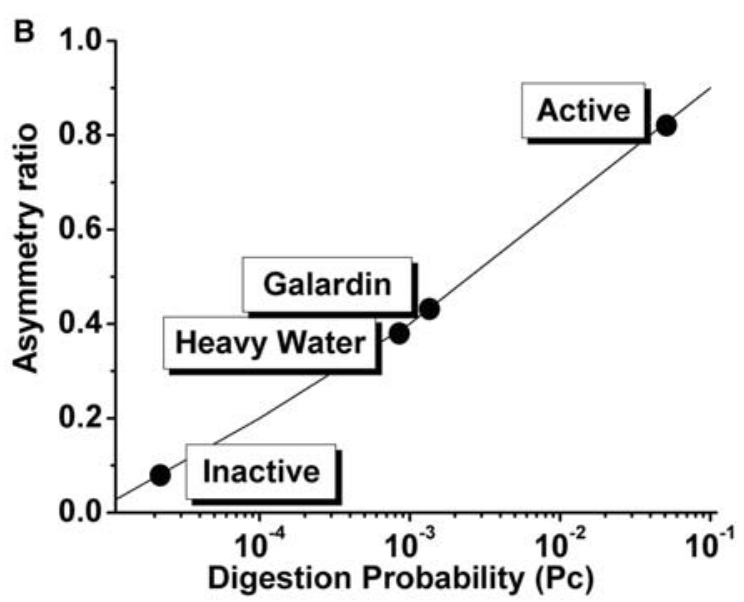

fusion plus flow or 1D diffusion models, respectively. They are in good agreement with experimental functions obtained for WT and inactive mutant enzymes. (A, inset) The simulated concentration profiles of WT (dashed line) and inactive mutant (solid line) enzymes on a collagen fibril. The simulation was composed as described above. The concentration profile was summed over $24 \mathrm{~s}$ of the simulation. (B) Monte Carlo simulations were performed as in Fig. $3 \mathrm{~B}$ with a reflective boundary condition set in the middle of the fibril representing the no-transport block. The number of molecules on each side of the block was recorded to calculate the asymmetry ratios as in Fig. 2. The simulations demonstrate that the asymmetry ratio is highly dependent on the value of $P_{C}$. The asymmetry ratios obtained experimentally with active MMP-1, its inactive mutant, and MMP-1 inhibited by the protease inhibitor Galardin, or heavy water, are indicated. 
depends on unfolding of a collagen fibril that can become rate-limiting. The activation energy for fibril digestion (101 kcal $\mathrm{mol}^{-1}$ ) was found to be 4 times that of a helical collagen monomer (26). The high energy of activation for collagenolysis is in good agreement, however, with the apparent energy of activation for collagen fibril unfolding $\left(124 \mathrm{kcal} \mathrm{mol}^{-1}\right)$ measured more recently (27).

We thus conclude that the MMP-1collagen system is a Brownian ratchet that is able to rectify Brownian forces into a propulsion mechanism by coupling to collagen proteolysis. The minimum power of $1.7 \times 10^{-19} \mathrm{~W}$ per molecule of MMP-1 is required to propel the enzyme along the collagen fibril with the velocity $V=4.5 \pm$ $0.36 \mu \mathrm{m} \mathrm{s}^{-1}$, which was calculated from the diffusion coefficient measured experimentally (15). A maximum power input for the directional transport of MMP-1 at room temperature $\left(P_{\mathrm{C}}=10 \%\right)$ of $1.1 \times 10^{-18} \mathrm{~W}$ per molecule of MMP-1 was calculated from the assumption of $5 \mathrm{kcal} / \mathrm{mol}$ of free energy for a cleaved peptide bond (15). The efficiency of the motor at room temperature can then be calculated as the ratio of power output over input to be $15 \%$ (28). These numbers are expected to change as the value of $P_{C}$ increases with rise of the temperature.

The biological consequences of the collagenase motor activity are of great interest. We hypothesize that the collagenase ratchet can serve as a clutch mechanism, assisting cell locomotion on collagen matrices and contraction of collagen gels in three-dimensional cultures. Association of the enzyme with cell membranes via interaction with tissuespecific integrins can couple the extracellular proteolysis with the forces exerted by the cytoskeleton to direct membrane protrusions along a "no-skid" surface generated by the digestion of collagen fibrils. Membrane-type MMPs $(29,30)$ can potentially act in a similar fashion. Experimental observations that clearly demonstrate the requirement of MMP-1-dependent collagenolysis for migration of keratinocytes on collagen support this hypothesis (31).

References and Notes

1. T. Vu, Z. Werb, Genes Dev. 14, 2123 (2000).

2. M. Egeblad, Z. Werb, Nature Rev. Cancer 2, 161 (2002).

3. K. E. Kadler, D. F. Holmes, J. A. Trotter, J. A. Chapman, Biochem. J. 316, 1 (1996).

4. R. Visse, H. Nagase, Circ. Res. 92, 827 (2003).

5. G. I. Goldberg et al., J. Biol. Chem. 261, 6600 (1986).

6. G. Fields, H. Van Wart, H. Birkedal-Hansen, J. Biol. Chem. 262, 6221 (1987).

7. R. P. Feynman, R. B. Leighton, M. Sands, The Feynman Lectures on Physics (Addison-Wesley. Reading, MA, 1966), vol. 1.

8. R. D. Astumian, Science 276, 917 (1997).

9. F. Jülicher, A. Ajdari, J. Prost, Rev. Mod. Phys. 69, 1269 (1997).

10. C. Peskin, G. Odell, G. Oster, Biophys. J. 65, 316 (1993).

11. J. Prost, J. F. Chauwin, L. Peliti, A. Ajdari, Phys. Rev. Lett. 72, 2652 (1994).
12. R. F. Fox, Phys. Rev. E 57, 2177 (1998).

13. J. Mai, I. M. Sokolov, A. Blumen, Phys. Rev. E. 64 011102 (2001).

14. J. Torbet, M. Ronziere, Biochem. J. 219, 1057 (1984).

15. Materials and methods are available as supporting material on Science Online.

16. S. Saffarian, E. L. Elson, Biophys. J. 84, 2030 (2003).

17. D. Magde, E. L. Elson, W. W. Webb, Biopolymers 17, 361 (1978).

18. M. Schliwa, G. Woehlke, Nature 422, 759 (2003).

19. R. D. Vale, R. A. Milligan, Science 288, 88 (2000).

20. H. Qian, Biophys. Chem. 83, 35 (2000).

21. L. J. Windsor, M. K. Bodden, B. Birkedal-Hansen, J. A. Engler, H. Birkedal-Hansen, J. Biol. Chem. 269, 26201 (1994).

22. D. L. Steele et al., Protein Eng. 13, 397 (2000).

23. I. E. Collier, S. Saffarian, B. L. Marmer, E. L. Elson, G. Goldberg, Biophys. J. 81, 2370 (2001).

24. S. Saffarian, I. E. Collier, B. L. Marmer, E. L. Elson, G. Goldberg, unpublished observations.

25. J. J. Jeffrey, H. G. Welgus, R. Burgeson, A. Eisen, J. Biol. Chem. 258, 11123 (1983).

26. H. Welgus, J. Jeffrey, G. Stricklin, W. Roswit, A. Eisen, J. Biol. Chem. 255, 6806 (1980).
27. E. Leikina, M. V. Mertts, N. Kuznetsova, S. Leikin, Proc. Natl. Acad. Sci. U.S.A. 99, 1314 (2002).

28. I. Derényi, M. Bier, R. D. Astumian, Phys. Rev. Lett. 83, 903 (1999).

29. T. Shimada et al., Eur. J. Biochem. 262, 907 (1999).

30. E. Ohuchi et al., J. Biol. Chem. 272, 2446 (1997).

31. B. K. Pilcher et al., J. Cell Biol. 137, 1445 (1997).

32. The authors would like to thank M. S. Conrady for the use of the superconducting magnet. We also thank H. Qian and A. Eisen for reading the manuscript and helpful discussions. This work was supported in part by NIH grants GM-38838 to E.L.E., AR40618, AR39472 to G.I.G. and Washington University-Pfizer Inc. agreement.

Supporting Online Material

www.sciencemag.org/cgi/content/full/306/5693/108/ DC1

Materials and Methods

SOM Text

Figs. S1 and S2

Tables S1 to S3

References and Notes

15 April 2004; accepted 5 August 2004

\title{
Life History Trade-Offs Assemble Ecological Guilds
}

\begin{abstract}
Michael B. Bonsall, ${ }^{1 *} \dagger$ Vincent A. A. Jansen, ${ }^{2 *}$ Michael P. Hassell ${ }^{1}$
Ecological theory predicts that competition for a limiting resource will lead to the exclusion of species unless the within-species effects outweigh the betweenspecies effects. Understanding how multiple competitors might coexist on a single resource has focused on the prescriptive formalism of a necessary niche width and limiting similarity. Here, we show how continuously varying life histories and trade-offs in these characteristics can allow multiple competitors to coexist, and we reveal how limiting similarity emerges and is shaped by the ecological and evolutionary characteristics of competitors. In this way, we illustrate how the interplay of ecological and evolutionary processes acts to shape ecological communities in a unique way. This leads us to argue that evolutionary processes (life-history trait trade-offs) are fundamental to the understanding of the structure of ecological communities.
\end{abstract}

Ecological theory predicts that species in ecological communities can coexist only if there are differences in their responses to limiting resources $(1,2)$. Evolutionary processes underpin this coexistence: Differences between species arise through the combined effects of selection and life-history tradeoffs. Trade-offs in life histories $(3,4)$ prevent species from evolving as Darwinian "demons" (species that develop rapidly, reproduce continuously, and do not age). Sanctioning investment in life-history characteristics against ecological competitive ability has the potential to allow multiple species to coexist by reducing the probability that any one species is demonic in an assemblage (5-9). Reducing the dominance of any one species in an assemblage and

\footnotetext{
${ }^{1}$ Department of Biological Sciences, Imperial College London, Silwood Park Campus, Ascot, Berkshire SL5 7PY, UK. ${ }^{2}$ School of Biological Sciences, Royal Holloway University of London, Egham, Surrey TW20 OEX, UK.

*These authors contributed equally to this work. $\dagger$ To whom correspondence should be addressed. E-mail: m.bonsall@imperial.ac.uk
}

fostering equivalence among species has the potential to allow ecological diversity to be promoted $(10,11)$.

Here, we explore the hypothesis that communities are assembled or shaped through evolutionary processes and that diversity is maintained and generated through species equivalence. Using a continuously varying strategy set, we evaluate the role of assembly and trade-offs as mechanisms for the formation of complex predator-prey assemblages. We consider a generic interaction between a class of natural enemies (parasitoids) competing for a limiting host resource $(12,13)$. We assume that $n$ types of consumer can attack particular juvenile (larval) stages of the host and that there is no a priori partitioning of this resource class. We describe the interaction between competing parasitoids within a generation with a system of differential equations. Solutions to these equations provide a description of the population dynamics of the system from which (ecological) difference equations (14) can be derived. Finally, the invasion and replacement 